\title{
Editorial
}

\section{Sesenta años de las revistas de psicología en Colombia*}

\author{
Julio César Ossa y Jean Nikola Cudina \\ Universidad de San Buenaventura (Colombia)
}

Referencia formato APA: Ossa, J. C., \& Cudina, J. N. (Ed.). (2016). Sesenta años de las revistas de psicología en Colombia. Rev. Guillermo de Ockham, 14(2), 7-17. doi: http://dx.doi.org/10.21500/22563202.2720

En el año de 1956, Mateo Mankeliunas fundó en la Universidad Nacional de Colombia la primera publicación especializada de la disciplina: Revista de psicología. Desde entonces han transcurrido sesenta años de producción científica y académica de la psicología en el país. Por tal razón, dedicamos esta editorial a los proyectos que han marcado y moldeado la historia de las publicaciones científicas de la psicología en Colombia. Empezaremos por caracterizar el devenir histórico de los proyectos editoriales de la psicología colombiana para llegar a un campo de tensión donde mostramos que esos proyectos -sesenta años después- quedan en un limbo.

\section{Tres periodos en la historia de las publicaciones en Colombia}

\section{Periodo de emergencia (1956-1976)}

Este periodo establece los cimientos de los proyectos editoriales de la psicología en Colombia. Algunos años despues de la primera edición de la Revista de Psicología surgieron cuatro revistas más, a saber: Archivos de estudios psicoanaliticos y de psicología médica (1964), Revista latinoamericana de psicología (1969), Revista de la Sociedad Colombiana de Psicoanálisis (1976) y Cuadernos de psicología (1976). ${ }^{1}$ Si bien esto implicó pronto el paso a un periodo fructífero de producción académica de la psicología colombiana, las publicaciones durante este periodo se caracterizaron por tener una periodicidad descontinua, con niveles precarios de calidad editorial y científica de acuerdo con los requerimientos mínimos de una publicación científica (Cano, 1995).

\section{Periodo de transicional (1977-1996)}

Durante este periodo emergieron múltiples proyectos editoriales, gran parte de ellos creados para divulgar los productos de los nacientes grupos de investigación (producción endogámica). Se crearon diecisiete revistas, pero solo seis lograron sobrevivir hasta la fecha. Las revistas que desaparecieron no alcanzaron más de cinco años de difusión académica y científica (Jaraba-Barrios, et al., 2011).

\section{Periodo de asentamiento (1997-2016)}

Esta fase, además de representar una época en la que los proyectos editoriales que emergieron se mantuvieron en una constante divulgación académica desde el momento de creación hasta la actualidad, es un momento en el que se dan a conocer las prácticas, políticas de evaluación y medición de las publicaciones seriadas del país ${ }^{2}$ y se le apuesta al mejoramiento de los criterios de calidad editorial y científica de las publicaciones académicas El desafío mayor será asumir el desarrollo de las prácticas editoriales en función de la internacionalización, la visibilidad y el impacto de la producción académica. Sin duda, ello ha dado a la producción académica de la psicología colombiana una significativa visibilidad en las comunidades académicas nacionales e internacionales en los últimos veinte años. Si bien los proyectos editoriales posteriores gozaron de mayor sistematicidad, no se puede desconocer que se beneficiaron de la tecnología y el internet para visibilizar sus productos. Otros factores que determinaron el panorama en lo que se ha llamado el periodo de transición, fueron la Ley 100 de la educación superior y las políticas de investigación de Colciencias. Esos factores, entre otros, jalonaron definitivamente el desarrollo de la

\footnotetext{
* Los autores agradecen a la Universidad de San Buenaventura Cali y a la Asociación Colombiana de Facultades de Psicología Ascofapsi la financiación del proyecto de investigación Estudio bibliométrico de la producción académica en Colombia. Agradecemos a Luis Merchán Paredes y a Martha Restrepo Forero por el respaldo brindado al grupo Bibliometrics.

1. En la actualidad, la Revista latinoamericana de psicología, fundada por Rubén Ardila, es la única que sobrevive a este periodo.

2. A finales del año 1996 Colciencias - por medio de Publindex- llevó a cabo la primera convocatoria dirigida a los editores de las revistas científicas del país, con el fin de recabar información que permitiera clasificar las revistas, lo cual constituye una práctica de evaluación hasta nuestro tiempo. 
producción científica y redundaron en el fortalecimiento de la disciplina en la comunidad académica de psicólogos en Colombia. Es así como en los últimos veinte años se concreta un trabajo formalizado que ha contribuido a una mayor presencia de la producción científica colombiana en las comunidades académicas nacionales e internacionales (Cudina \& Ossa, 2016).

\section{Qué tenemos ahora en Colombia}

Sesenta años de trabajo editorial en Colombia se pueden ver reflejados en la Tabla 1 de clasificación de Publindex, un sistema que determinaba calidad editorial y científica de las revistas con unos criterios propios.

Tabla 1

Clasificación de Revistas Psicología colombianas por el sistema de información permanente de Publindex, 2015

\begin{tabular}{ccc}
\hline Categoría & Número de revistas & Porcentaje \\
\hline A1 & 8 & $29,6 \%$ \\
\hline A2 & 4 & $14,8 \%$ \\
\hline B & 3 & $11,1 \%$ \\
\hline C & 7 & $25,9 \%$ \\
\hline-- & 5 & $18,5 \%$ \\
\hline Total & 27 & $100 \%$ \\
\hline
\end{tabular}

Fuente: Modelo de clasificación de revistas científicas (Publindex, 2015)

Según el Publindex, la categoría A supone que las revistas han logrado una alta calidad editorial y científica. Entre ellas encontramos ocho $(29,6 \%)$ con clasificación A1 (máxima categoría) y cuatro (14,8 \%) en A2. Las categorías $\mathrm{B}$ y $\mathrm{C}$ suponen un esfuerzo de las revistas por mejorar su calidad editorial. En este grupo encontramos tres revistas $(11,1 \%)$ en la categoría B y siete $(25,9 \%)$ en la categoría C. De acuerdo con esta clasificación, solo cinco revistas $(18,5 \%)$ no lograron indexarse en Publindex.

\section{El nuevo periodo de las revistas colombianas y la pérdida de autonomía de Publindex}

Con el nuevo modelo de medición y evaluación para la indexación de revistas científicas de Colombia, la historia de las publicaciones seriadas de la psicología en el país da un giro hacia un nuevo periodo. Según este nuevo modelo, ya no es suficiente sustentar la calidad editorial y científica, sino que es necesario demostrar el impacto de las publicaciones seriadas. Este nuevo periodo se instala a partir del fenómeno denominado la herida narcisista de las revistas de psicología, el cual explicamos a continuación.

La ilusión de la clasificación anterior se desvanece porque ninguna de las revistas A1 y A2 pudo sostener su clasificación, dado que desde el 2016 la clasificación de las revistas se determinará según su ubicación en los cuartiles (Q1, Q2, Q3 y Q4) del Journal Citation Report (JCR). Esta pérdida de autonomía de Publindex, cuyo propósito fundamental era la fusión de revistas, desplaza automáticamente las revistas A1 y A2 a las categorías B y C. Las demás que no se encuentran indexadas en Scopus o WoS deberán rasguñar una clasificación ( $\mathrm{B}$ o $\mathrm{C}$ ) de acuerdo con el índice $\mathrm{H} 5$, con los resultados arrojados por el Google Scholar (índice un tanto arbitrario por la heterogeneidad de los resultados que arroja y por la fijación abusiva de un periodo de cinco ańos). En la Tabla 2 se presenta la categorización de las revistas de acuerdo con el nuevo modelo de evaluación de Publindex.

Tabla 2

Resumen de categorización del nuevo modelo de medición de revistas

\begin{tabular}{cccc}
\hline Categoría & $\begin{array}{c}\text { Publindex } \\
\mathbf{2 0 1 5}\end{array}$ & $\begin{array}{c}\text { Nuevo } \\
\text { Publindex }\end{array}$ & $\begin{array}{c}\text { Ubicación de } \\
\text { cuartiles }\end{array}$ \\
\hline A1 & 8 & - & Q1 (JCR) \\
\hline A2 & 4 & - & Q2 (JCR) \\
\hline B & 3 & 4 & Q3 (JCR) o Q1 (H5) \\
\hline C & 7 & 3 & Q4 (JCR) o Q2 (H5) \\
\hline- & 5 & $20^{*}$ & \\
\hline
\end{tabular}

* Son veinte las publicaciones académicas de la psicología colombiana que no se encuentran incluidas en los índices del Jornal Citation Report. Para tal fin, la medida de impacto se determinará a partir del H5 arrojado por Google Scholar y para ello Publindex ha fijado unos umbrales que determinarán los cuartiles correspondientes a cada disciplina. No obstante, a la fecha no se conocen los umbrales del nuevo modelo.

Pero el problema de este nuevo modelo atañe no solo a Colombia, sino a toda la región latinoamericana y europea, toda vez que las revistas con mayor factor de impacto se concentran en EE. UU. y el Reino Unido. La industria editorial en la actualidad produce mayores ingresos que la industria automotriz (Ordónez-Matamoros, Cozzens \& García-Luque, 2011) y está monopolizada por dos grandes casas editoriales: Elsevier y Springer. Una publicación Q1 va desde los 1.350 dólares (PlosOne) hasta los 2.400 (Frontiers) y los investigadores (y algunas instituciones) pagan no solo porque suponen que el factor de impacto se transferirá - por defecto-a su publicación, sino también porque el sistema educativo colombiano se dejó seducir por la visibilidad y el alto impacto que ofrecen las vitrinas del mercado editorial internacional. Veamos cómo esos ingresos económicos se distribuyen por región geográfica (Tabla 3). 
Tabla 3

Clasificación por cuartiles de las revistas de psicología incluidas en el JCR

\begin{tabular}{lcccccc}
\hline \multicolumn{1}{c}{ Países } & Q1 & Q2 & Q3 & Q4 & Total & Porcentaje \\
\hline Latinoamérica & & 1 & 13 & 24 & 38 & $3,6 \%$ \\
\hline Estados Unidos & 169 & 127 & 106 & 56 & 459 & $43,4 \%$ \\
\hline Inglaterra & 81 & 93 & 62 & 29 & 267 & $25,2 \%$ \\
\hline Resto del mundo & 25 & 36 & 85 & 151 & 295 & $28 \%$ \\
\hline Total general & 275 & 257 & 266 & 260 & 1058 & $100 \%$ \\
\hline
\end{tabular}

Fuente: Elaboración propia con datos del JCR - 2016

El panorama que surge requiere una discusión con la demanda que genera la empresa científica, más aún en un periodo en el que los procesos de cambio y las dinámicas de producción académica en Colombia se encuentran en mutación. Lo alarmante es apoyarse en un modelo de medición asimétrico para determinar la calidad editorial de las publicaciones académicas de un país que no tiene la estructura económica ni las instituciones que apoyen su crecimiento.

Ahora el desafío es mayor. Implica necesariamente reconocer el papel de las publicaciones seriadas del país como canales de comunicación científico-académica y de relevancia social. Las lógicas de evaluación determinadas en el impacto de los índices internacionales de citación nos muestran una producción académica local y regional ampliamente invisibilizada. Por ello, la responsabilidad de Colciencias a través de Publindex debe ser impostergable: garantizar la visibilidad de la producción científica de las comunidades académicas y certificar una estructura editorial que posibilite el acompañamiento en la indización de las publicaciones seriadas en WoS y Scopus (Aguilar, 2016). Ello sin descuidar el valor y el compromiso que traen consigo las revistas emergentes comprometidas con la visibilidad y el impacto de la producción académica local, regional y nacional.

\section{Referencias}

Aguilar, M. C. (Ed.). (2016). Los cambios en el modelo de medición Publindex: nuevos retos, nuevas exigencias. $\mathrm{Di}$ versitas, 12(2), 163-164.

Cano, V. (1995). Characteristics of the publishing infrastructure of peripheral countries: a comparison of periodical publications from Latin America with periodicals from the US and the UK. Scientometrics, 34(1), 121-138.

Cudina, J. N., \& Ossa, J.C. (2016). The top 100 highimpact papers in Colombian psychology: a bibliometric study from WoS and Scopus. Informação \& Sociedade: Estudos, 26(2), 137-154.

Jaraba-Barrios, B., Guerrero-Castro, J., Gómez-Morales, Y. J., \& López-López, W. (2011). Bibliometría e historia de las prácticas académicas locales: un esbozo a partir del caso de la psicología en Colombia. Avances en Psicología Latinoamericana, 29(2), 354-369.

Ordóńez-Matamoros, G., Cozzens, S.E., García-Luque, M. (2011). North-South and South-South research collaboration: What differences does it make for developing countries? - The case of Colombia. Trabajo presentado en Atlanta Conference on Science and Innovation Policy: Building Capacity for Scientific Innovation and Outcomes. GA: United States. 